\title{
Allitridin inhibits human cytomegalovirus replication in vitro
}

\author{
JU ZHANG, HUI WANG, ZHI-DAN XIANG, SAI-NAN SHU and FENG FANG \\ Department of Pediatrics, Tongji Hospital, Tongji Medical College, \\ Huazhong University of Science and Technology, Wuhan, Hubei 430032, P.R. China
}

Received October 23, 2012; Accepted February 5, 2013

DOI: $10.3892 / \mathrm{mmr} .2013 .1328$

\begin{abstract}
Human cytomegalovirus (HCMV) has been associated with a wide spectrum of diseases. There is currently no effective treatment for eliminating the virus. Garlic bulb extract has been reported to possess anti-viral efficacy. This study aimed to investigate the expression of the immediate-early (IE; ul122 and ul123), early (E; ul54) and late (L; ul83) genes of HCMV as well as the inhibitory effect of allitridin on the transcription levels of these genes. The results indicated that a HCMV gene expression cascade occurred, and that the deletion of IE72 had no influence on the transcription of the ul122 gene, while it led to significant reductions of ul54 and ul83 mRNA expression levels. Additionally, allitridin effectively suppressed the transcription of the HCMV IE, E and L genes; the inhibition rates of the transcription of the ul122 and ul123 genes were higher compared with those of ul54 and ul83 mRNA expression, while the expression of the IE genes was not significantly reduced by ganciclovir (GCV). Our results indicate that the HCMV IE72 deletion mutant strain affects the transcription of the virus downstream gene, allitridin inhibits HCMV infection in vitro, and that the IE genes may be the key target of allitridin in its action against HCMV.
\end{abstract}

\section{Introduction}

Human cytomegalovirus (HCMV) is a large double-stranded DNA virus of the human herpesvirus family; it belongs to the $\beta$-herpesviridae subfamily of the herpes viruses. HCMV is a significant human pathogen which infects the majority of the world's population (1). Although HCMV infection in adults and healthy children is usually asymptomatic, it causes severe disease, including retinitis, pneumonitis, hepatitis, gastroenteritis and end-organ disease, in immunodeficient and/or immunocompromised individuals, such as organ transplant patients, AIDs patients, congenitally infected neonates and cancer patients

Correspondence to: Professor Feng Fang, Department of Pediatrics, Tongji Hospital, Tongji Medical College, Huazhong University of Science and Technology, 1095 Liberation Avenue, Wuhan, Hubei 430032, P.R. China

E-mail: ffang@tjh.tjmu.edu.cn

Key words: human cytomegalovirus, allitridin, gene, mRNA, $\triangle$ IE72 strain, SYBR real-time PCR undergoing chemotherapy (2,3). Kenneson and Cannon (4) have reported that the rates of congenital CMV infection are between 0.3 and $6.2 \%$ of all live births in developed countries.

Since there is no vaccine for HCMV, antiviral drugs constitute the current treatment for CMV infection; the available antiviral drugs include ganciclovir (GCV), valganciclovir, foscarnet, cidofovir and fomivirsen $(5,6)$. However, these antiviral therapies have limited efficacy and a high incidence of side-effects. Therefore, it is necessary to develop a novel drug of low toxicity and high efficiency for the treatment of HCMV infection. Garlic bulb has long been used as a natural fungicide, and it is also known as a 'natural antibiotic'. Allitridin is an active constituent of garlic bulbs, and a number of studies have demonstrated its pharmaceutical effects (7-9). These studies have shown that allitridin has a variety of actions potentially useful for human health, such as lowering hepatic cholesterol storage, detoxification, anti-oxidant, antifungal and antibacterial activities, as well as tumor suppression and prevention of heart disease $(10,11)$. The antiviral effects of allitridin against HCMV have also been shown in previous studies (12).

A previous study performed in our laboratory demonstrated that allitridin reduces viral loads in the liver and improves the histopathological lesions and functions of the liver (13). Another, more recent study by our group has shown that allitridin treatment reduces viral levels in the salivary glands and facilitates the healing of pathologic lesions in organs, particularly during the chronic infection phase, and that it has mild anti-MCMV properties in vivo (14). To further assess the target and mechanisms of action of allitridin against HCMV, immediate-early (IE; ul122 and ul123), early (E; ul54) and late (L; ul83) gene transcription was investigated following the treatment of HCMV-infected cells with allitridin or GCV. SYBR real-time PCR was used to analyze the expression levels of the mRNAs of these genes in various treatment groups; the HCMV IE72 deletion mutant was evaluated as a control in order to investigate the inhibitory effect of allitridin against IE72 as well as the synergistic effect of IE72 and IE86. Finally, we aimed to ascertain whether allitridin inhibits the expression of IE genes and then the expression of $\mathrm{E}$ and $\mathrm{L}$ genes, to confirm the main link of the antiviral mechanism and to provide an experimental basis for its anti-HCMV therapeutic effects.

\section{Materials and methods}

Cell culture and virus. Human embryo lung fibroblast (HEL) cells were cultured in Dulbecco's modified Eagle's medium 
(DMEM; Gibco, Carlsbad, CA, USA) supplemented with 10\% (v/v) heat-inactivated fetal calf serum (FCS), $100 \mathrm{U} / \mathrm{ml}$ penicillin and $100 \mu \mathrm{g} / \mathrm{ml}$ streptomycin in a humidified incubator at $37^{\circ} \mathrm{C}$ in the presence of $5 \% \mathrm{CO}_{2}$. The HCMV AD169 strain was provided by the Institute of Virology, Chinese Academy of Preventive Medical Sciences (Beijing, China) and the HCMV IE72 deletion mutant strain ( $\triangle \mathrm{IE} 72$ ), which is obtained by deletion of exon 4 from the major IE gene and does not express IE72 protein, was provided by M Zhang (Medical College of Georgia, Augusta, GA, USA). The mean infective viral quantities of AD169 and $\triangle \mathrm{IE} 72$ were $\sim 4.56 \times 10^{6}$ and $3.52 \times 10^{6} \mathrm{PFU} /$ $\mathrm{ml}$, respectively. The multiplicity of infection (MOI) is the viral infectious titer divided by the cell count; a high MOI $(\mathrm{MOI}=2.5)$ was used in this experiment. HEL cells were digested with $0.20 \%$ pancreatic enzyme and seeded in 6-well plates at a concentration of $2 \times 10^{5} / \mathrm{ml}$; the monolayer cells were then infected with the appropriate amount of HCMV AD169 or $\Delta$ IE72 strain. The starting time $(0 \mathrm{~h})$ was after $1 \mathrm{~h}$ of virus adsorption, and normal cells were evaluated as the control. Following 0.5, 2, 4, 6, 12 and $24 \mathrm{~h}$ of infection, the cells were harvested, and maintained in refrigerators at $-80^{\circ} \mathrm{C}$ until use.

Drugs. Allicin injection was purchased from Shanghai Harvest Pharmaceutical Co., Ltd. (cat. no. 100801, 15 mg/ml; Shanghai, China). GCV injection was obtained from Hubei Keyi Pharmaceutical Co., Ltd. (cat. no. 100714; Hubei, China).

Cytotoxicity assay. The MTT assay was used to determine the cytotoxicity of allitridin to HEL cells. HEL cells were cultured in a 96-well plate in the absence or presence of various concentrations of allitridin; each concentration of allitridin was used in 4 wells. Following a 72-h culture, the plates were read at $560 \mathrm{~nm}$ in a microplate reader. The percentage of surviving cells in each test group was then calculated and the maximum tolerated concentrations (MTCs) of allitridin and GCV in the HEL cells were determined to be 9.6 and $2.3 \mu \mathrm{g} / \mathrm{ml}$, respectively.

Establishment of the infected cell model. HEL cells were seeded in 6-well plates at a concentration of $2 \times 10^{5} / \mathrm{ml}$, and the monolayer cells were then infected with the appropriate amount of HCMV AD169 or $\triangle \mathrm{IE} 72$ strain. The viruses were adsorbed for $1 \mathrm{~h}$ and the end of the viral adsorption process was considered to be the zero point of the time course. The nutrient medium was then changed to a drug-free (with $4 \%$ FCS) or drug-containing $(9.6 \mu \mathrm{g} / \mathrm{ml}$ allitridin or $2.3 \mu \mathrm{g} / \mathrm{ml} \mathrm{GCV})$ maintenance medium. In this experiment, the cell groups were: i) the experimental group, HCMV AD169 strain-infected cells treated with allitridin; ii) the control groups: a) HCMV AD169 strain-infected cells; b) HCMV AD169 strain-infected cells treated with GCV; c) HCMV $\triangle$ IE72 strain-infected cells; d) HCMV $\triangle$ IE72 strain-infected cells treated with allitridin; and e) HCMV $\triangle$ IE72 strain-infected cells treated with GCV. Each culture was harvested 0.5, 2, 4, 6, 12 and $24 \mathrm{~h}$ post-infection (p.i.) separately and maintained at $-80^{\circ} \mathrm{C}$ until RNA extraction was conducted.

Transcriptional level analyis of the HCMV-related genes. Total RNA extraction was performed on these samples using the TRIzol reagent (Invitrogen Life Technologies, Carlsbad,

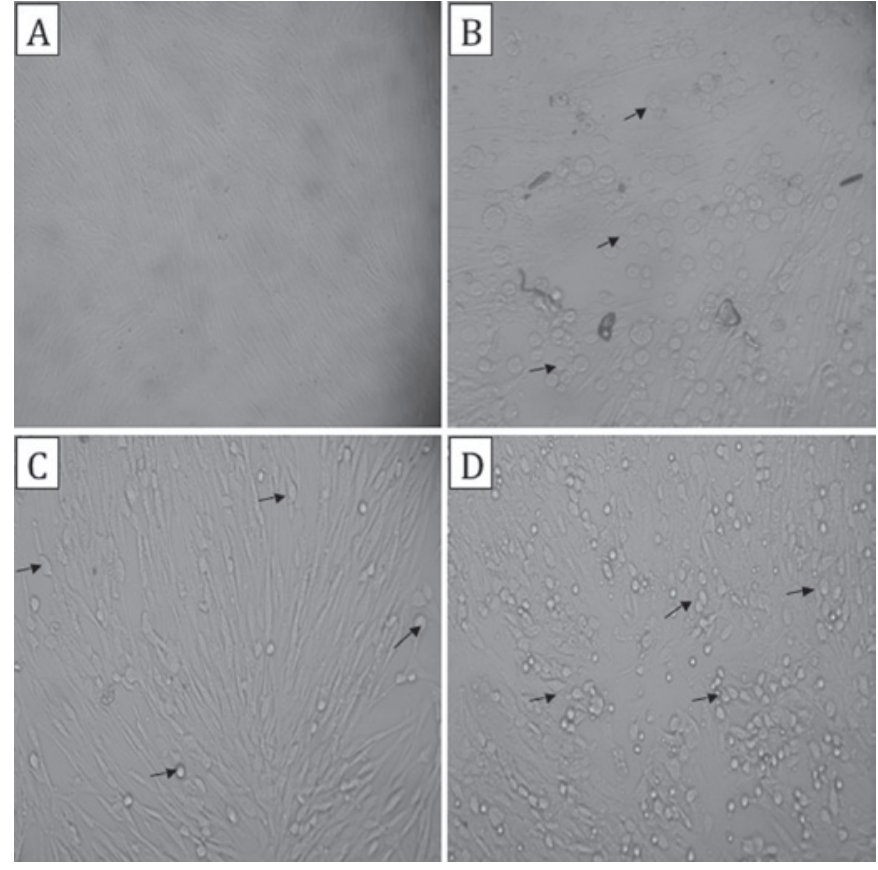

Figure 1. Observation of cells from four of the groups (magnification, x200). (A) Normal HEL cells; (B) following allitridin addition, the HEL cells contracted and the gaps between cells increased; (C) HEL cells were clearly swollen (bigger and rounder) $24 \mathrm{~h}$ after infection with AD169; (D) allitridin-treated HEL cells were more markedly swollen and most cells shrank and became round; a disordered arrangement of cells was observed and there were some floating cells $24 \mathrm{~h}$ after infection.

CA, USA) following the manufacturer's instructions. To exclude cellular DNA contamination, the samples were treated with DNase I (Qiagen, Hilden, Germany). Dry RNA pellets were dissolved in nuclease-free water and the concentration was estimated using a NanoDrop ND-1000 Spectrophotometer (Nano Drop Technologies, Inc., Wilmington, DE, USA). cDNA was synthesized from $300 \mathrm{ng}$ RNA using Prime-Script ${ }^{\mathrm{TM}}$ RT Enzyme Mix I (Takara Bio, Inc, Shiga, Japan). In each cDNA sample, the target and reference genes were detected simultaneously by SYBR real-time PCR using the Mx3000 $\mathrm{P}^{\circledR}$ real-time PCR system (Stratagene, La Jolla, CA, USA). The primers of the IE, E and L genes of HCMV were as follows: ul122 (78 bp) forward, 5'-ATCATGCTGCCCCTCATC AAA-3' and reverse, 5'-GATAATCTTGTTGCGGTACTGGAT-3; ul123 (86 bp) forward, 5'-GCTCCTCTGATTCTCTGGTGTC-3' and reverse, 5'-ACTGTTCTCAGCCACAATTACTG-3'; ul54 (148 bp) forward, 5'-GACCTATTCGTTTTCACACCTACG-3' and reverse, 5'-ATACTGTAGCCGTGTTCTGTGG-3'; ul83 (159 bp) forward, 5'-GCAGCCACGGGATCGTACT-3' and reverse, 5'-GGCTTTTACCTCACACGAGCATT-3'. The human glyceraldehyde phosphate dehydrogenase (GAPDH, $92 \mathrm{bp)}$ gene was used as an amplification control. The GAPDH primers were: forward, 5'-GGTTTATGGAGGTCCTCT TGTGT-3' and reverse, 5'-AACTACCCATGACTCAGC TTCTC-3'. SYBR real-time PCR and melting curve analysis were performed using the SYBR ${ }^{\circledR}$ Premix Ex Taq ${ }^{\mathrm{TM}}$ II (Perfect Real-Time; Takara Bio, Inc.) according to the manufacturer's instructions. The standard curves for the real-time PCR were generated using HCMV DNA. The amounts of HCMV mRNA were quantified relative to GAPDH expression. 
A
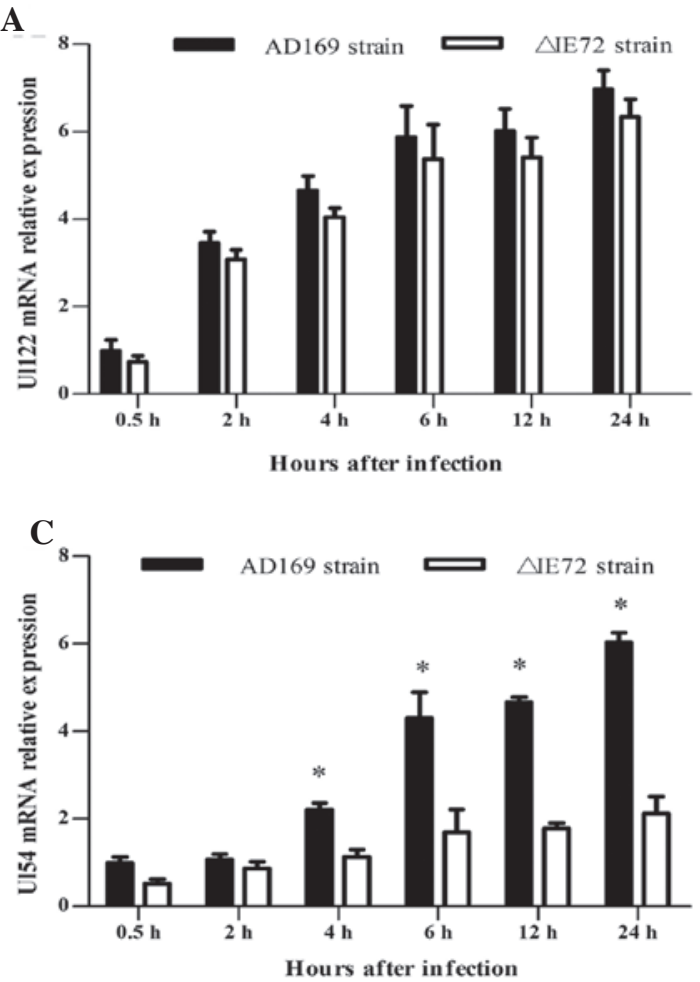
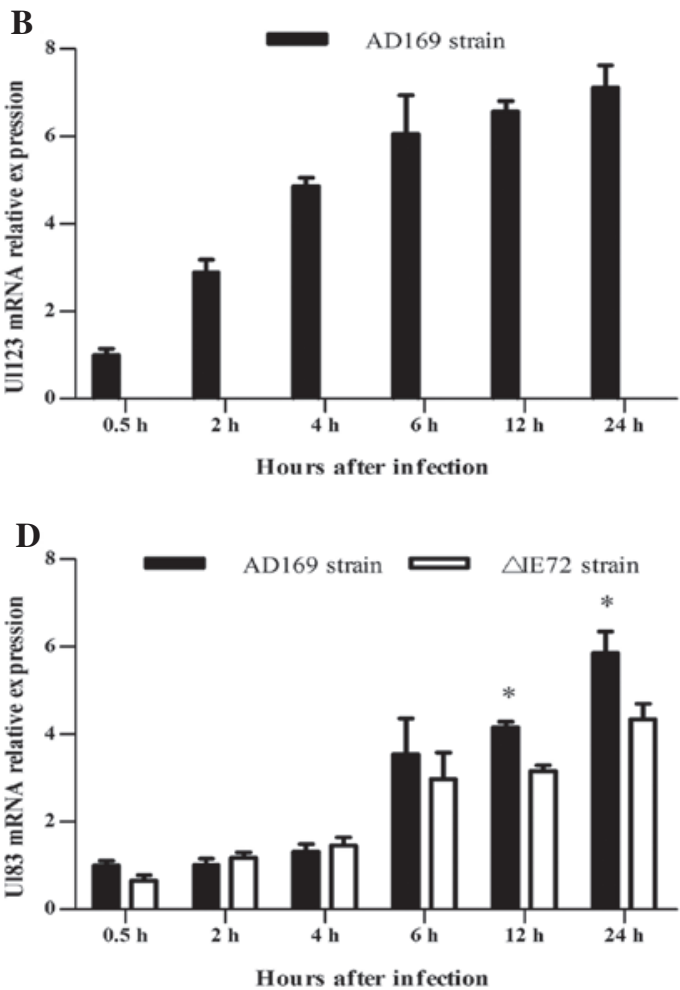

Figure 2. HCMV-related gene mRNA expression in the AD169 and $\Delta \mathrm{IE72}$ strain-infected groups. (A) ul122, (B) ul123, (C) ul54 and (D) ul83. Data are expressed as the mean \pm standard error of the mean $(\mathrm{SEM})$. ${ }^{*} \mathrm{P}<0.05$ using Student's t-test.

Statistical analysis. The $2^{-\Delta \Delta C T}$ method was used to investigate the different transcriptional levels of the IE, E and L genes of $\mathrm{HCMV}$, as well as the inhibitory effects of drugs against the HCMV-related genes (15). All the tests were repeated three times. The data were analyzed using SPSS 15.0 software and expressed as the mean \pm standard error of the mean (SEM). Comparisons between two groups were by analysis of variance and statistical differences between groups were determined using the Student's t-test. All tests were two-sided and $\mathrm{P}<0.05$ was considered to indicate a statistically significant difference.

\section{Results}

Observation of cells in each group. Allitridin is a potent irritant to HEL cells. When the cell morphology was observed under an inverted microscope, cell contraction and gaps between the cells were noted following the addition of allitridin, but the cell morphology soon recovered in the infected HEL cells. Lesions were observed in the cells of the HCMV AD169 strain-infected control and allitridin- or GCV-treated groups $8 \mathrm{~h}$ p.i. More specifically, the cells were swollen, had increased in size and become rounder in shape, and were arranged in a disorderly manner. Twenty-four hours p.i., the cells of the AD169-infected control and GCV-treated groups remained markedly swollen. In the allitridin-treated group, some of the cells remained markedly swollen, however, most of the cells had decreased in size. Moreover, the arrangement of cells was disordered and there were some floating cells. Lesions were also observed in the cells of the HCMV $\triangle \mathrm{IE} 72$ strain-infected control and allitridin- or GCV-treated groups $16 \mathrm{~h}$ p.i., including swelling and a disorderly arrangement (Fig. 1).
HCMV gene expression. The expression pattern of HCMV genes described in the present study is consistent with that reported in previous studies (16). Fig. 2 shows the gene expression in HEL cells following HCMV infection. The expression pattern of ul122 mRNA in the cells of the HCMV AD169 strain-infected groups was similar to that in the cells of the HCMV $\triangle \mathrm{IE} 72$ strain-infected groups (Fig. 2A). The expression began quickly and the levels rapidly increased following infection in the two virus-infected groups. The transcription of the ul123 gene in the HCMV AD169 strain-infected cells rose from 0.5 to $6 \mathrm{~h}$ p.i. and then increased modestly from 6 to 24 h p.i. (Fig. 2B). However, there was no ul123 gene expression in the HCMV $\triangle \mathrm{IE} 72$ strain-infected cells. The ul54 mRNA levels in the two types of virus-infected cells were low as infection began; however, the expression level in the AD169 strain-infected cells was clearly increased and continued to increase $4 \mathrm{~h}$ p.i., while in the HCMV $\triangle I E 72$ strain-infected cells it increased slowly and the growth rate was significantly lower than that in the HCMV AD169 strain-infected cells $(\mathrm{P}<0.05$; Fig. 2C). The expression pattern of ul83 in the HCMV AD169 strain-infected cells was similar to that in the HCMV $\triangle$ IE72 strain-infected cells (Fig. 2D). The expression levels in the two types of virus-infected cells were low during the first $4 \mathrm{~h}$ p.i. but significantly increased $6 \mathrm{~h}$ p.i. The expression level of ul83 mRNA in the $\Delta$ IE72-infected cells was lower than that in the AD169-infected cells $6 \mathrm{~h}$ p.i. The levels of ul83 mRNA expression in the two types of virus-infected cells were significantly different at the last two time points $(\mathrm{P}<0.05)$.

Allitridin significantly suppresses the expression of HCMV IE genes. The present study demonstrated that the expression levels of the ul122 gene of the two virus strains in the 

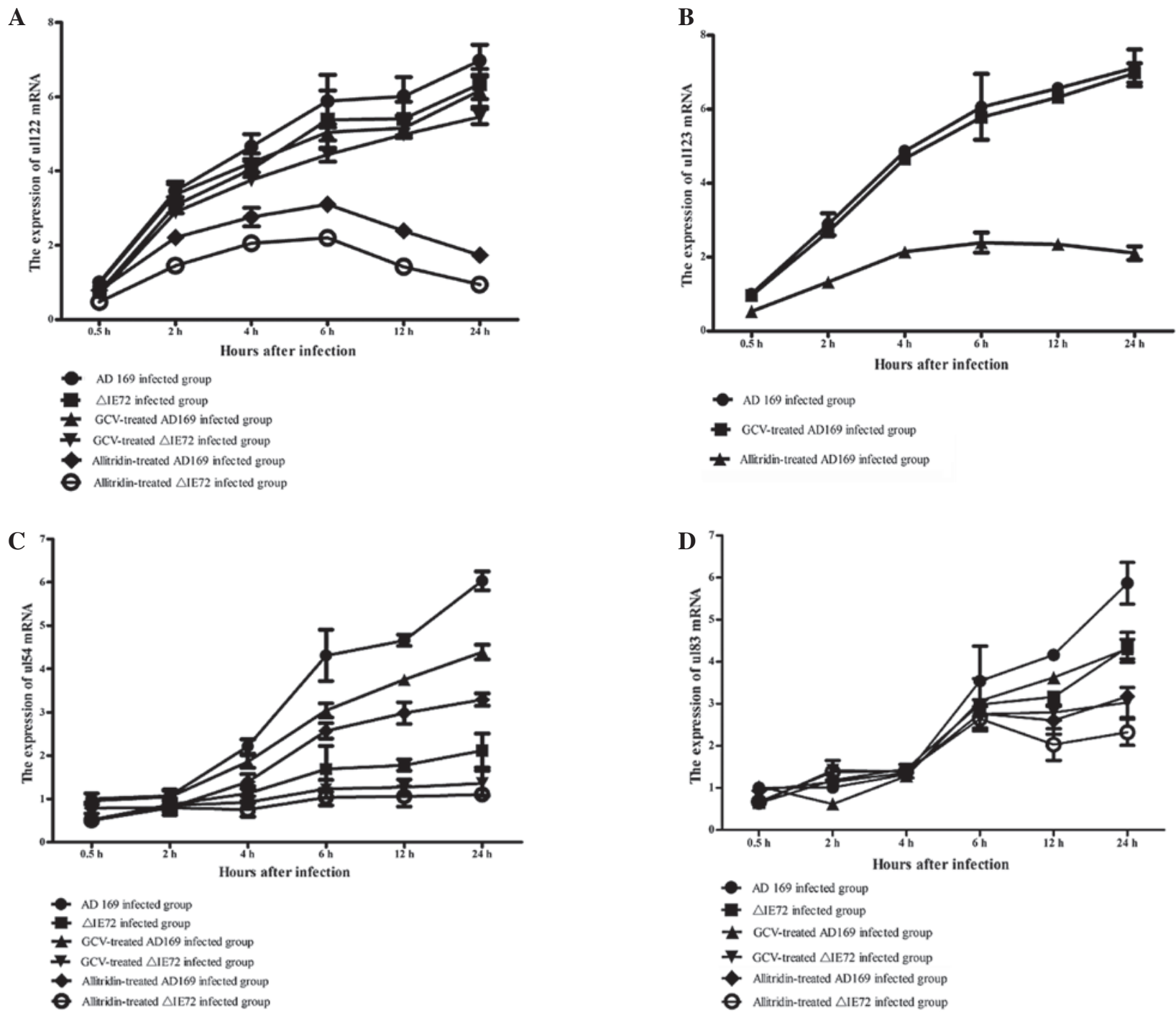

Figure 3. Effects of allitridin and CGV against HCMV IE, E and L gene expression. HEL cells infected with HCMV AD169 and $\triangle \mathrm{IE72}$ strains at a multiplicity of 2.5 and treated with allitridin or GCV. The mRNA levels were semi-quantified using real-time PCR. Effect of allitridin on HCMV (A) ul122; (B) ul123; (C) ul54 and (D) ul83 genes. Data are expressed as the means \pm SEM. P $<0.05$ using Student's t-test. GCV, ganciclovir; HCMV, human cytomegalovirus; IE, immediate-early; E, early; L, late.

allitridin- and the GCV-treated cells were lower than those in the virus-infected control groups (without drug treatment) following infection (Fig. 3A). The expression levels of the ul122 gene in the allitridin-treated cells were significantly lower than those in the GCV-treated and virus-infected control groups $(\mathrm{P}<0.01)$. The ul122 mRNA expression levels were significantly decreased in the AD169-and $\triangle$ IE72-infected cells following treatment with allitridin, and were lower in the $\Delta$ IE72-infected cells. In the AD169-infected cells, the expression of the ul123 gene following allitridin treatment was significantly lower than that in the AD169-infected control cells $(\mathrm{P}<0.01$; Fig. 3B), while there was no difference between the GCV-treated and the AD169-infected control groups $(\mathrm{P}>0.05)$. In the $\Delta \mathrm{IE} 72$-infected cells, the expression of ul123 mRNA was not detected since exon 4 of the ul123 coding region was knocked down (Fig. 3B).

Allitridin suppresses the expression of the HCMV E gene. The expression levels of the AD169 ul54 mRNA in the allitridin- and GCV-treated cells were lower than those in the virus-infected control cells (Fig. 3C), and the differences between the expression levels were statistically significant $(\mathrm{P}<0.05)$. The expression levels of the $\triangle \mathrm{IE} 72 \mathrm{ul} 54 \mathrm{mRNA}$ in the allitridin- and the GCV-treated cells were also lower than those in the virus-infected control cells. The inhibitory effects of allitridin and GCV against HCMV ul54 mRNA were more potent in the $\triangle$ IE72-infected cells than in the AD169-infected cells (Fig. 3C).

Allitridin suppresses the expression of the HCMV L gene. During the first $6 \mathrm{~h}$ following HCMV infection, the expression levels of the ul83 gene were not different among the six groups $(\mathrm{P}>0.05)$. However, $6 \mathrm{~h}$ following infection, the expression levels of the ul 83 gene in the drug-treated groups (GCV or allitridin) were lower than those in the virus-infected control groups, whether infected by the AD169 or the $\triangle \mathrm{IE} 72$ strain. A more potent inhibitory effect was observed in the allitridin-treated cells 12 and 24 h p.i. $(\mathrm{P}<0.05)$, particularly in the allitridin-treated $\triangle \mathrm{IE} 72$ strain-infected cells, when compared with the GCV-treated cells (Fig. 3D). 


\section{Discussion}

The results of the present study regarding the expression of HCMV genes were consistent with the results of previous studies, and indicate that a gene expression cascading effect occurred (16). The HCMV gene family includes IE, E and L genes. The IE genes encode immediate-early antigens (IEAs), mainly containing IE72 (ul122) and IE86 (ul123) proteins, which have the highest expression levels and the broadest range of functional activities. The $\mathrm{E}$ gene encodes early antigens (EAs) which are enzymes and regulatory factors essential for the synthesis of progeny DNA and proteins, such as the viral DNA polymerase (UL54). UL54 is the catalytic subunit of HCMV DNA polymerase, and the expression of ul54 gene is mainly regulated by IE proteins (IE 72 and IE 86). Late antigens (LAs) are viral structural proteins, such as the capsule matrix proteins (UL83, pp65) (17). In the present study, the HCMV $\triangle$ IE72 strain was used as a control virus strain in order to investigate the inhibitory effect of allitridin against IE72, the synergistic action of IE72 and IE86, and the regulation of the transcription of ul54 and ul83 by IE72. The $\Delta$ IE72 strain was not able to express IE72 since the exon 4 of the ul123 coding region was knocked down. At a low MOI, only a small amount of IE86 was expressed, while the early and late proteins were not expressed. Thus, the $\Delta$ IE72 strain was not able to replicate, proliferate and form plaques (18). Therefore, the $\triangle$ IE72 strain and adenovirus Ad5-infected HEK cells (adenovirus packaging cell) were simultaneously used, since the cofactor required for the proliferation of the $\Delta \mathrm{IE} 72$ strain was provided by Ad5 (19).

In the present study, it was found that the expression level of ul122 mRNA in the two virus strains was similar; it was not influenced by the deficiency of IE72, in accordance with the findings of a previous study (20). When treated with allitridin and GCV, the expression levels of ul122 mRNA in the two types of virus strain-infected cells were decreased, while the reduction in the GCV-treated cells was lower than that in the allitridin-treated cells. Notably, the inhibitory effect of allitridin against the types of two virus-infected cells increased in a time-dependent manner, and was significantly higher in the HCMV $\triangle \mathrm{IE} 72$ strain-infected cells. The difference between the effects of allitridin on the expression of the ul122 genes of the two viruses suggests that allitridin may affect additional links, such as other virus IE proteins, to inhibit the expression of ul122 mRNA. Moreover, this may also be associated with the regulatory function of IE86. IE86 acts alone or in synergy with IE72 to trans-activate the IE promoter; it may also be combined with a DNA regulatory sequence, such as the TATA box upstream of main immediate early genes (MIE), to realize its own negative feedback control $(21,22)$. We hypothesized that the significant inhibitory effect of allitridin against the transcription of $\Delta \mathrm{IE} 72$ strain ul122 is associated with several reasons: the strong and long-lasting inhibitory effect of allitridin against the transcription of ul122, the $\Delta$ IE72 strain lacks the synergy of IE72, and the regulatory mechanism of IE86 when challenged by drug exposure is not able to compensate for or resist the inhibitory effect of allitridin. We subsequently analyzed the expression of the HCMV ul123 gene. It was observed that the expression of ul123 mRNA in the allitridin-treated cells was significantly lower than that in the control cells in the HCMV AD169 strain-infected groups. The expression of ul123 mRNA was not detected in the $\Delta$ IE72-infected cells since the exon 4 of the ul123 coding region was knocked down. Our results demonstrated that allitridin clearly inhibits the expression of HCMV ul123 mRNA, while there was no significant difference between the ul123 mRNA levels in the GCV-treated and control cells.

These results indicate that the inhibitory effect of allitridin on HCMV IE gene expression began early and was prolonged. It was also observed that allitridin inhibited the expression of ul122 mRNA to a greater extent than ul123 mRNA ( $24 \mathrm{~h}$ p.i.), which was consistent with the results of our previous study (23). Consequently, whether at the transcriptional or the protein level, the inhibitory effect of allitridin on IE86 was greater than that on IE72, while the differences between the inhibitory effects at the DNA and protein levels indicated that the drug is effective at the transcriptional, post-transcriptional, translational and post-translational levels. The IE86 protein is essential since it creates a favorable cellular environment for viral replication; the MIE gene locus consists of an enhancer-containing promoter upstream of the IE1 (ul123) and IE2 (ul122) genes (24). Our results indicated that allitridin had a significant inhibitory effect against the transcription of ul122 and ul123, which may occur during the transcription process of the MIE gene to the precursor RNA. There were no significant differences between these two genes in the GCV-treated and the control cells, suggesting that the IE genes may not be the key targets of GCV against HCMV.

The product of ul54 mRNA is the catalytic subunit of HCMV DNA polymerase (symbolic early gene), which interacts with UL44 (the auxiliary subunit of HCMV DNA polymerase) through the $\mathrm{C}$ terminal of its polypeptide chain, is phosphorylated and then has enzymatic activity (25). Our results demonstrated that the level of expression of the ul54 gene in the $\Delta \mathrm{IE} 72$-infected cells was lower than that in the AD169-infected cells, indicating that the deletion of IE72 resulted in a reduction in ul54 mRNA expression, and that the transcription of the virus downstream genes may be affected by IE72 deletion. It was noted that the expression levels of AD169 and $\triangle$ IE72 ul54 mRNA in the drug-treated cells were lower than those in the virus-infected control cells; the expression level in the $\Delta \mathrm{IE} 72$-infected cells was lower, so it was concluded that the inhibitory effects of allitridin and GCV against $\triangle \mathrm{IE72}$ ul54 mRNA were stronger. The inhibitory effects of allitridin against the ul54 mRNA of the two virus strains were significantly lower than those against ul122 and ul123 mRNA. This may be due to the fact that the expression of the ul54 gene is regulated by the IE protein, suggesting that the IE genes may be the key functional link between the actions of allitridin. Compared with GCV, allitridin significantly inhibited the expression of IE genes, and subsequently affected the expression of the E gene. When compared with IE genes, GCV can inhibit the E gene more markedly, which is consistent with the mechanism of GCV which is the competitive inhibition of viral DNA polymerase.

pp65 (u183) often serves as one of the target proteins in the clinical diagnosis of HCMV active infection due to its high levels of expression. Previous studies have shown that the pp65 protein is an important protein which takes part in viral gene expression and regulation, altering the metabolism of host cells 
and viral replication (17). Our results showed that the expression level of ul83 mRNA in the $\triangle I E 72$-infected cells was lower than that in the AD169-infected cells $6 \mathrm{~h}$ p.i., suggesting that the deletion of IE72 also affects the expression of the virus L gene. The reduction was not as marked as that of the $\mathrm{E}$ gene of the $\triangle \mathrm{IE} 72$ strain; this may be due to the fact that the synthesis of late virus protein occurs after the replication of viral genes, and the expression may be influenced by additional factors. Following treatment with allitridin or GCV, the expression of ul83 mRNA was clearly decreased compared with that in the respective control group 12 and 24 h p.i. Furthermore, the inhibitory effect of allitridin against the $\mathrm{L}$ gene was similar to that against the E gene, while the affects against both were lower than those against the IE genes. Since the expression of HCMV genes occurred in a sequential cascade, IE genes may be the key sites at which allitridin acts. While GCV had no inhibitory effect on IE genes but inhibited E (ul54) and L genes (ul83), this was consistent with its main mechanism, the inhibition of DNA polymerase activity.

It was hypothesized that allitridin significantly inhibits the expression of HCMV IE genes (ul122 and ul123) at the transcriptional level. The inhibitory effects against the two IE genes were similar, which indicates that the action of allitridin against the transcription of IE genes affected the progress of the transcription of the MIE gene to the precursor RNA. By using the $\triangle \mathrm{IE} 72$ strain as a control, it was observed that the expression of ul122 and ul123 mRNA have a clear synergistic association. The inhibitory effects of allitridin against the $\triangle$ IE72 strain ul122, ul54 and ul83 mRNA were higher than those against the respective mRNA of the AD169 strain. The degree of inhibition decreased in the order ul122 mRNA $>$ ul54 mRNA $>$ ul83 mRNA, indicating that there were many factors involved in the regulation of the upstream to downstream gene transcription and the regulation of the expression of the HCMV IE, E and L genes. Following analysis of the degree of inhibition of allitridin against ul54 and ul83 mRNA, as well as the influence of IE72 deficiency on the expression of ul122, ul54 and ul83 mRNA, it was hypothesized that the inhibitory action of allitridin against the expression of ul54 and ul83 mRNA may occur indirectly through the inhibition of the expression of IE gene mRNA. It is well known that GCV acts by inhibiting the viral DNA polymerase and incorporates viral DNA directly to terminate the extension of the viral DNA chain (26). In the replication cycle of HCMV, the expression of IE protein occurs prior to the replication of viral DNA and is followed by the expression of the E gene (u154), which encodes DNA polymerase. Following completion of the DNA replication, the $\mathrm{L}$ gene begins to express encoded virus structural proteins and the replication cycle is then finished. Therefore, the effect of GCV was consistent with its mechanism. The observation that the inhibitory effect of GCV against HCMV was different from that of allitridin, and less potent, demonstrates that the antiviral mechanisms of GCV and allitridin are different.

In conclusion, the present study showed that the expression pattern of related gene mRNAs in the AD169 strain-infected cells was similar to the pattern in the $\Delta$ IE72 strain-infected cells, and that the deletion of IE72 affects the expression of virus downstream genes. Following drug treatment, it was concluded that allitridin significantly inhibits the transcription of HCMV-related genes, and that IE genes constitute the main target; the key link may be the progress of the MIE gene transcript to the precursor RNA. This may provide a theoretical basis for the effect of allitridin against HCMV infection, and support the advantageous antiviral properties of traditional Chinese medicine. Since circumstances did not allow the investigation of the cytotoxicity of allitridin in vivo, the concentration of allitridin in the blood is not known. Future studies will aim to focus on the treatment of infections caused by HCMV and the investigation of additional potential inhibitory pathways.

\section{Acknowledgements}

This study was supported by a grant (no. 30070928) from the National Natural Science Foundation of China, awarded to Feng Fang.

\section{References}

1. Cannon MJ, Schmid DS and Hyde TB: Review of cytomegalovirus seroprevalence and demographic characteristics associated with infection. Rev Med Virol 20: 202-213, 2010.

2. Chan G, Nogalski MT, Stevenson EV and Yurochko AD: Human cytomegalovirus induction of a unique signalsome during viral entry into monocytes mediates distinct functional changes: a strategy for viral dissemination. J Leukoc Biol 92: 743-752, 2012.

3. Crough $\mathrm{T}$ and Khanna R: Immunobiology of human cytomegalovirus: from bench to bedside. Clin Microbiol Rev 22: 76-98, 2009.

4. Kenneson A and Cannon MJ: Review and meta-analysis of the epidemiology of congenital cytomegalovirus (CMV) infection. Rev Med Virol 17: 253-276, 2007.

5. Santos LF and Carratalà J: Treatment of cytomegalovirus disease. Enferm Infecc Microbiol Clin 29: 65-69, 2011.

6. Pescovitz MD: Valganciclovir: recent progress. Am J Transplant 10: 1359-1364, 2010.

7. Powolny AA and Singh SV: Multitargeted prevention and therapy of cancer by diallyl trisulfide and related Allium vegetable-derived organosulfur compounds. Cancer Lett 269: 305-314, 2008.

8. Durak I, Kavutcu M, Aytac B, Avci A, Devrim E, Ozbek H and Ozturk S: Effects of garlic extract consumption on blood lipid and oxidant/antioxidant parameters in humans with high blood cholesterol. J Nutr Biochem 15: 373-377, 2004.

9. Leuschner RGK and Ielsch V: Antimicrobial effects of garlic, clove and red hot chilli on Listeria monocytogenes in broth model systems and soft cheese. Int J Food Sci Nutr 54: 127-133, 2003.

10. Lu Y, He Z, Shen X, Xu X, Fan J, Wu S and Zhang D: Cholesterol-lowering effect of allicin on hypercholesterolemic ICR mice. Oxid Med Cell Longev 2012: 489690, 2012.

11. Agarwal KC: Therapeutic actions of garlic constituents. Med Res Rev 16: 111-124, 1996.

12. Guo NL, Lu DP, Woods GL, Reed E, Zhou GZ, Zhang LB and Waldman RH: Demonstration of the anti-viral activity of garlic extract against human cytomegalovirus in vitro. Chin Med J 106: 93-96, 1993.

13. Liu ZF, Fang F, Dong YS, Li G and Zhen H: Experimental study on the prevention and treatment of murine cytomegalovirus hepatitis by using allitridin. Antiviral Res 61: 125-128, 2004.

14. Liu XL, Wang H, Li YN, Ge HX, Shu SN and Fang F: Effects of allitridin on acute and chronic mouse cytomegalovirus infection. Arch Virol 156: 1841-1846, 2011.

15. Livak KJ and Schmittgen TD: Analysis of relative gene expression data using real-time quantitative PCR and the $2^{-\Delta \Delta C T}$ method. Methods 25: 402-408, 2001.

16. Murphy E and Shenk T: Human cytomegalovirus genome. In: Human Cytomegalovirus: Current Topics in Microbiology and Immunology. Shenk TE and Stinski MF (eds). Springer, New York, NY, pp.1-19, 2008.

17. White EA, Del Rosario CJ, Sanders RL and Spector DH: The IE2 60-kilodalton and 40-kilodalton proteins are dispensable for human cytomegalovirus replication but are required for efficient delayed early and late gene expression and production of infectious virus. J Virol 8125: 2573-2583, 2007. 
18. Gawn JM and Greaves RF: Absence of IE1 p72 protein function during low-multiplicity infection by human cytomegalovirus results in a broad block to viral delayed-early gene expression. J Virol 76: 4441-4455, 2002.

19. Wilkinson GW and Akrigg A: Constitutive and enhanced expression from the CMV major IE promoter in a defective adenovirus vector. Nucleic Acids Res 20: 2233-2239, 1992.

20. Greaves RF and Mocarski ES: Defective growth correlates with reduced accumulation of a viral DNA replication protein after low-multiplicity infection by a human cytomegalovirus iel mutant. J Virol 72: 366-379, 1998.

21. Arlt H, Lang D, Gebert S and Stamminger T: Identification of binding sites for the 86-kilodalton IE2 protein of human cytomegalovirus within an IE2-responsive viral early promoter. J Virol 68: 4117-4125, 1994.

22. Ahn JH and Hayward GS: The major immediate-early proteins IE1 and IE2 of human cytomegalovirus colocalize with and disrupt PML-associated nuclear bodies at very early times in infected permissive cells. J Virol 71: 4599-4613, 1997.
23. Zhen H, Fang F, Ye DY, et al: Experimental study on the action of allitridin against human cytomegalovirus in vitro: inhibitory effects on immediate-early genes. Antiviral Res 72: 68-74, 2006.

24. Stinski MF and Petrik DT: Functional roles of the human cytomegalovirus essential IE86 protein. Curr Top Microbiol Immunol 325: 133-152, 2008.

25. Aitken C, Barrett-Muir W, Millar C, et al: Use of molecular assays in diagnosis and monitoring of cytomegalovirus disease following renal transplantation. J Clin Microbiol 37: 2804-2807, 1999.

26. De Clercq E and Neyts J: Antiviral agents acting as DNA or RNA chain terminators. In: Antiviral Strategies. Kräusslich HG and Bartenschlager R (eds). Springer, New York, pp.53-84, 2008. 